\title{
Design and Implementation of a Database for Special Agricultural Tracing System
}

\author{
Shanhong Zhu ${ }^{1,2}$, Lei Zhang ${ }^{1}$ \\ 1) School of Computer and Information Engineering, Xinxiang University, Henan, China \\ ${ }^{2)}$ International School of Software, Wuhan University, Wuhan, China
}

\begin{abstract}
In the paper we analyze the current lack of food security problems and solutions, and develope a SSH-based system under the wireless Internet of Thing eggs tracing system, in which the database is a very important part. The system uses SQLServer 2000 as its back-end database, employs MyEclipses Java Enterprise 10 as design environment, uses SSH as framework, uses RFID reading the tag, We also expound the design scheme of the system and the key technologies used ,improve system performance, provide an idea for the development of similar database module editors.
\end{abstract}

Keywords - Internet of things, Egg Tracing, Database, RFID

\section{一种特色农产品溯源系统数据库的设计和实现}

\author{
朱珊虹 ${ }^{1,2}$ 张碚 $^{1}$ \\ 1) 新乡学院信息工程学院, 新乡, 河南, 中国 \\ 2) 武汉大学国际软件学院, 武汉, 湖北, 中国
}

摘 要 分析当前食品安全问题以及解决方法上的不足, 开发了 SSH 架构下基于无线物联网技术的鸡蛋溯源系统, 数据库在其中 是一个非常重要组成部分, 系统以 SQLServer2000 作为后台数据库, 采用 MyEclipes Java Enterprise 10 为设计环境, 基于 SSH（Struts 2,spring2.5, hibernate 3.2)框架, 使用 RFID 读取器读取电子标签, 并详细阐述了数据库的设计方案和关键技术, 提高了系统的性能, 为 开发类似的数据库模块提供思路。

关键词 物联网, 鸡蛋溯源, 数据库, SSH, RFID

\section{1. 引言}

食品行业发展已经逐渐细化, 并趋向于全球化贸易模 式和生产方式专业化, 供应食品的渠道也日益复杂。食品 安全涉及到生产、加工、存储、运输、销售等整个食品的 方方面面 ${ }^{[1]}$ 。食品从最初的原料加工到最终消费者手里所 经历的环节越来越多, 究其原因, 一个方面, 从生产到消 费, 所经历的中间环节的增加, 一定程度上增加了引发食 品安全问题的概率; 另一个方面, 食品供应商和销售商的 增多, 一些不良商家在利益的驱动下, 选择不利于社会发 展的行为, 使得食品安全问题层出不穷, 并导致进一步恶 化。近年来发生了众多食品安全问题, 从三聚氰胺奶粉到 苏丹红鸡蛋、从双汇瘦肉精到西瓜膨大素, 以及最近发生

河南省科技厅项目（基金号：132300410079，1333020005）
的枸杞硫磺熏制事件, 都无一例外的说明中国严重的安全 问题, 其中有些因为无法快速准确的溯源而导致安全问题 不能得到及时有效的解决。因此, 建立一个科学的管理产品 生产、流通、销售过程的体系, 对预防食品安全事件的发 生具有非常重要的意义。

\section{2. 溯源系统概述}

本文以一种特色农产品一 “养子沟” 鸡蛋为例, 因为 河南特色 “养子沟” 鸡蛋被誉为“鸡蛋中的人参”, 具有“三 高一低”的特点: 高碘、高硒、高锌, 低胆固醇等特点, 不 仅有极高的营养价值和独特的风味, 而且易于吸收消化, 被推广和接收, 本研究在大量收集整理鸡蛋生产经营数据 和分析影响鸡蛋质量安全因素的基础上, 开发出基于物联 网的鸡蛋溯源系统, 该系统以监控视频的方式对鸡蛋的生 
产过程进行全程监控并保存入视频数据库, 同时平台通过 无线物联网技术对鸡蛋的生产过程进行全程记录, 采集的 生产信息也将存储在平台的强大数据库中, 便于统一管理 和综合查询, 平台还将在鸡蛋的运输和超市销售环节搭建 基于无线物联网的信息采集系统, 对鸡蛋产品从鸡场生产 环节、运输和销售全程追踪, 追踪信息同样存储在平台强 大的数据库中。最后, 消费者将能够通过手机客户端、定 点追溯终端或者网络平台对鸡蛋表面的 “二维码” 贴标进 行扫描, 进而查看鸡蛋的生产视频和全程运输细节数据, 让消费者更加明白所购买鸡蛋的来源和销售过程。

近年来, J2EE 的轻量级开源框架发展良好, 本系统数 据库采 用 SSH (Struts2、Hibernate、Spring) 架 构, 它是 一个开源的框架, 具有三层 MVC 结构, 便于开发, 层次 清晰。业务层采用 Spring 来管理, 在数据持久层采用 Hibernate 进行对象关系映射, 通过 Struts2 对 MVC(Model View Control)框架进行实现, 成功对各层次与模块解耦, 并控制表示层 JSP 页面的跳转工作 ${ }^{[2]}$ 。该系统前台客户端 程序主要负责数据采集、录入和管理, 后台 服 务 器主要 用于接收数据和实现管理等功能。系统拓扑结构如图 1

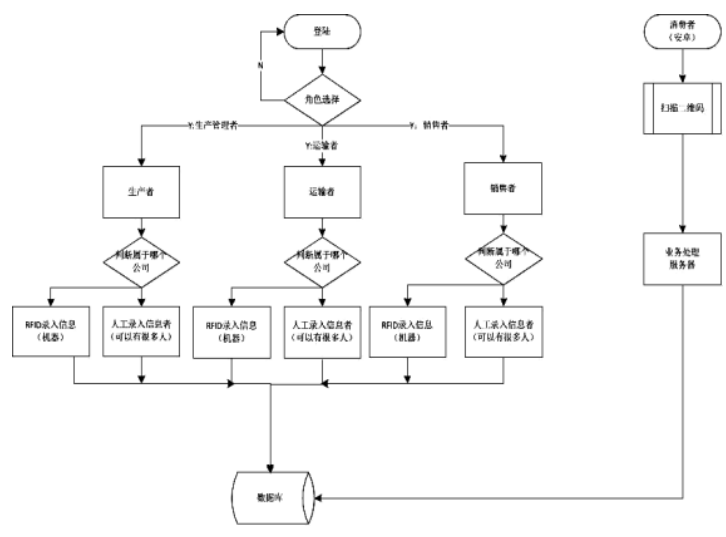

图 1 溯源系统拓扑结构图

在本系统中, 每个节点都有各自独立的服务器, 存储事 件数据, 而本地服务器中存储着 $\mathrm{EPC}$ 编码和服务器地址的 映射信息,同时将 EPC 编码与本地服务器地址的映射信息 存储到平台根服务器中, 通过这种方式在平台综合查询管 理系统中, 访问根服务器, 获取 EPC 编码对应的本地服务 器地址, 再获取 EPCIS 服务器地址, 并通过该地址访问 EPCIS 服务器, 获取产品的信息 ${ }^{[3]}$ 。数据库是本鸡蛋溯源系 统的核心, 数据采集设备将数据采集后存储到数据库中, 数 据库将其进行分类与归纳, 并进行实时存储, 为前台客户端 程序进行数据访问做好准备。

\section{3. 系统数据库分析}

数据库的设计是系统开发和实现的重要组成部分, 首 先要先从整体上对系统进行需求分析, 其次要从结构上准 确定位, 使其符合规范性, 通过概念设计、逻辑设计、物 理设计和验证设计建立一个完整的数据库。并不断进行调 整和维护以达到准确、完整的存储数据。本节将从以下需 求分析、系统结构对数据库进行分析。

\section{1 需求分析}

通过调查和分析, 本系统依据物联网和多种技术手段, 充分结合了特色鸡蛋现状和产品质量安全溯源系统的特 点, 既要具有鸡蛋生产、销售的信息, 还要包括运输、销售 的查询和消费者的查询和交流等功能。因此本系统综合客 户的业务活动和数据的使用情况, 研究数据的种类、范围、 数量以及它们在业务活动中交流的情况, 在保证其自身的 规范性要求的前提下满足系统的整体要求。本系统的数据 库数据有鸡蛋的生产视频及数据采集数据、鸡蛋生产物联 网数据采集数据、生产环节数据管理、运输环节物联网采 集数据、销售环节物联网采集数据、销售环节数据管理等, 功能模块如图 2 所示, 具体如下:

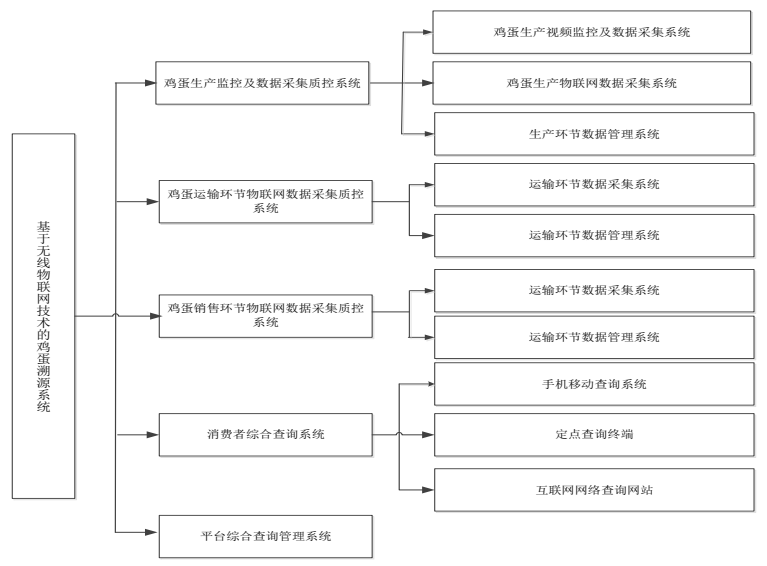

图 2 系统功能表

1. 鸡蛋的生产视频及数据采集系统, 系统将对鸡蛋的 各个生产环节进行视频采集, 并 且通过一系列复杂的存储 过程对采集的视频信息进行存储，并且方便消费者通过服 务器进行视频的观看和调用;

2. 鸡蛋生产物联网数据采集系统, 系统将通过无线物 联网技术对鸡蛋生产的各个细节，包括细节名称，时间， 人员等信息进行全面记录，方便消费者查询;

3. 生产环节数据管理系统, 系统主要职责是提供给鸡 蛋生产商管理工作人员查看鸡蛋 生产环节的具体信息, 并 且提供一定权限的修改和删除权限, 可以对鸡场生产环节 
进行全面追踪和监控。

4. 运输环节物联网数据采集质控系统, 系统将通过无 线物联网技术对运输环节的各个 细节, 包括细节名称, 时 间, 人员等信息进行全面记录, 方便消费者查询;

5. 运输环节数据管理系统, 系统主要职责是提供给鸡 蛋运输商管理工作人员查看鸡蛋 运输环节的具体信息, 并 且提供一定权限的修改和删除权限, 可以对鸡蛋运输环节 进行全面 追踪和监控。

6. 平台综合查询管理系统, 系统通过后台观看数据继 续数据库数据的直接查询、修改 和删除等操作, 维护数据 库正常运行。

\section{2 系统数据库结构分析}

本系统综合溯源系统的基本功能和数据库的标准规 范, 统一地设计该系统的属性数据和空间数据, 以及数据 库与数据之间的关系, 从而实现数据的无缝结合与共享。 本系统的数据库本身是相对独立的, 这样既保证了数据安 全性, 又能增加其自身拓展性, 为本系统的后期设计以及后 续新功能的开发提供支持[4]。

本系统应用中,数据库运行的主要过程是:RFID 读写器 读取 RFID 标签, 从中读取 $\mathrm{EPC}$, 然后读写器将读取到的 一连串 $\mathrm{EPC}$ 码以比特流的形式发送到后台服务器端 ${ }^{[5]}$ 。

RFID 读写器能读取带有 RFID 标签的物品, EPC 中间件 控制和管理各检测点的 RFID 标签和读写器等设备, 并向 $\mathrm{EPC}$ 物联网传输 EPC 信息, EPC 物联网将采集到的 EPC 信息保存到 EPCIS 服务器上。数据库不仅是提取数 据时的服务器端, 又是数据采集时的客户端。数据库是本 溯源系统中客户端程序与服务器程序存储数据的载体。后 台数据库管理主要负责记录和处理来自于以上环节的数据 和服务请求。以上的每一个环节都会包含各个环节的数据 管理系统, 以保证每个环节的数据完整正确的传送到后台 服务器中。溯源系统与数据库关系如图 3 所示:

\section{3 数据库冗余处理}

在本系统试行过程中发现, 虽然 RFID 读写器采集到 的标签数据量非常多, 但真正有实际用途的数据却不多。 因此在进行数据库设计时, 通常要采取数据规范化来减少 数据冗余, 如果不过滤掉冗余的数据, 会带来四个方面的 负担: 一是传输大量的标签数据, 会带来巨大的网络负荷; 二是建立过多的关联, 在表的插入、更新、删除操作时会 占用系统资源; 三是处理大量的标签数据, 会给数据服务 器带来负担; 四是存储额外的标签数据, 带来的大量存储 负担。因此本系统在 RFID 数据传递给系统之前对数据进

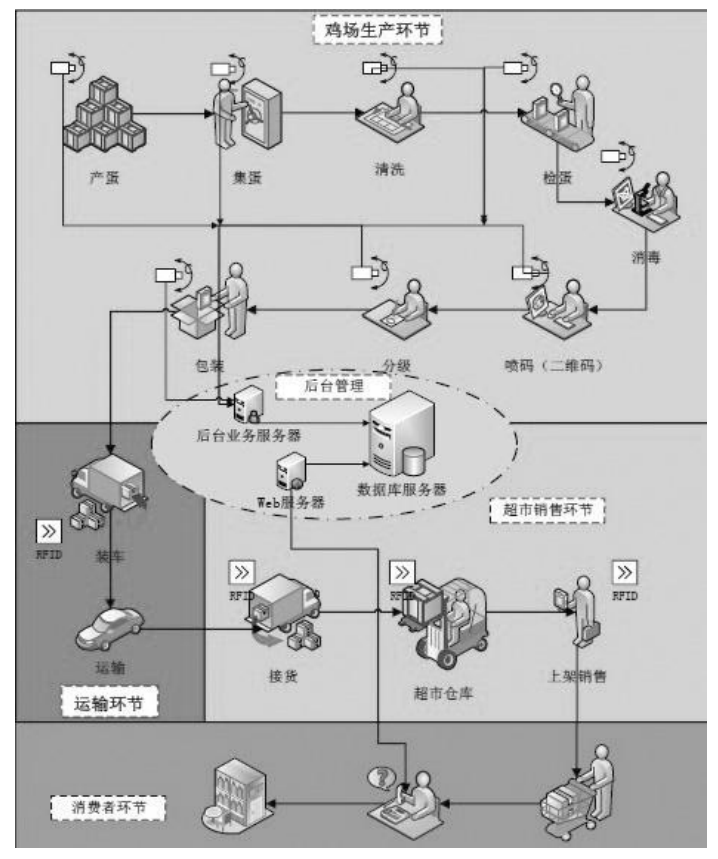

图 3 溯源系统与数据库关系图

行过滤。假定 RFID 读写器采集到的标签数据可以表示成 $<\mathrm{F}, \mathrm{P}, \mathrm{M}\rangle$, 其中读写器标识号为 F; 产品的标识码 $\mathrm{P} ; \mathrm{M}$ 表示标签的读取时间。过滤算法如下:

1) 将蛋类标签数据放入一个哈希表中, 将产品标识码 030302019908610000000000408220130810090099994 其 中 0303020199 字段是鸡蛋在国家统计局统计用产品的分 类号、第二个字段 086 是中国区代码、第三个字段 2013081009009999 是养殖场的营业执照号、第四个字段是 该养殖场给该鸡蛋编制的唯一的序列号、最末字段是校验 码), 作为哈希表的关键字。2)假设一个时间间隔 I(Interval) 如 $30 \mathrm{~s}$, 当读取到一个新的标签数据时, 扫描是否存在相同 的标签在哈希表中, 若检查到相同且两者的读取时间差 <I 时, 就认为该标签数据是重复的电子标签数据, 过滤掉的 同时更新该标签在哈希表中的读取时间。若存在且两者的 读取时间差>I 则认为该标签是新的标签, 输出该标签同时 更新哈希表中该标签的读取时间。若不存在, 则将其插入 到哈希表中同时输出该标签。

本系统数据库实现了数据标准化和规范化, 我们将基 本数据划分为养殖场信息、鸡蛋信息、质量信息、运输信 息、超市信息、人员信息等标签数据。指定这些信息的主 键分别为养殖场编号、鸡蛋编号、质量等级编号、运输编 号、超市编号、以及工作人员编号。每个电子标签有唯一 编号, 并且每条记录与记录时间有关, 所以标签数据的主键 为标签编号和记录时间的组合。将这些表中不依赖于主键 的数据属性去掉, 最后确定的数据库模型图如图 4: 


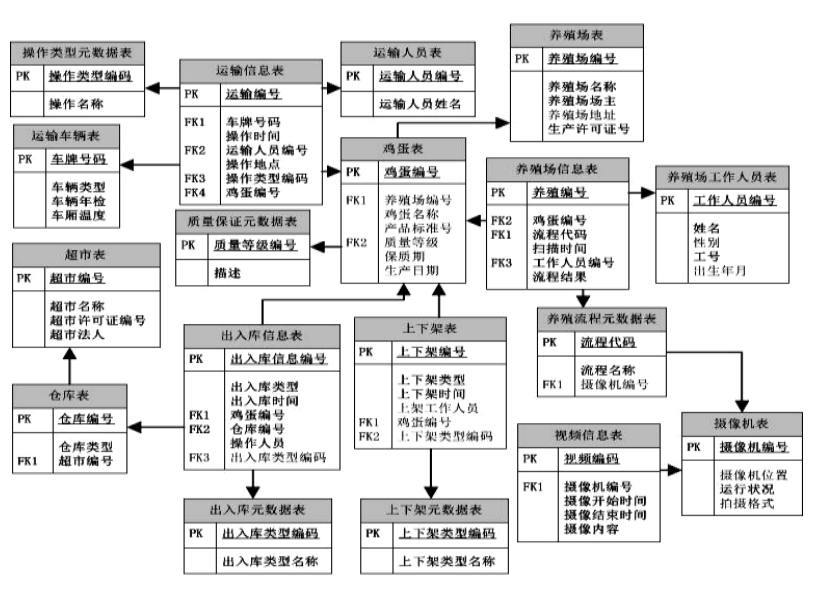

图4 鸡蛋溯源数据库模型图

\section{4. 结论}

食品安全是关系到人民的切身利益和国家的长治久 安, 本鸡蛋溯源系统是运用计算机技术和物联网技术实现 智能追溯的典型例子, 数据库在本系统中起到了重要作用, 由此提出的数据库设计方案, 从需求分析到结构分析等方 面设计出优化的数据库关系表, 并从技术方面介绍了数据 库系统的搭建和实现, 从而提高了系统的运行效率, 对进 一步在其他方面开发溯源系统提供了理论和数据支持。

\section{参考文献(References)}

[1] War qing, Li Sujian. Theory and application of radio frequency identification technology (RFID). Beijing: Electronic Industry Press, 2004: 140-144.

[2] Ren Shougang, Xu Huanliang. Based on RFID/GIS meat tracking and tracing system design and implementation Nuclear Science and Techniques.2010, (10): 229-234.

[3] Anderson, Joan G.ames L. Sea food quality: issues for consumer research. The Journal of Consumer Affairs, 1991, 25(1):144-16.

[4] Huang Ye China. Livestock agricultural products supply chain based on RFID information sharing research. South China University of technology, 2010.

[5] Jiao jie, zhao Yang Shu. Web access control system based on SSH framework implementation Journal of shenyang normal university (information science edition 2010, 28(4):507-509.

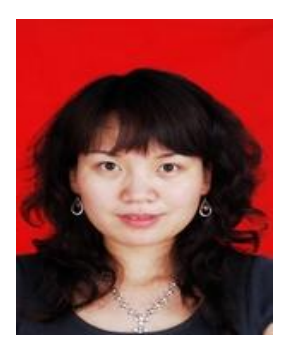

Shanhong Zhu (1982-) was born in China in 1982.She is working on towards $\mathrm{PhD}$ degree in the International School of Software at Wuhan University. She is employed as a Lecturer in Xinxiang College, where she is now a director in Computer Teaching and Research Department. Her research interests include computer multimedia, virtual reality application, embedded system and application, home automation, computer communication and so on. 